\title{
Positioning with Wide-Area GNSS Networks: Concept and Application
}

\author{
Tarig A. Ali
}

Department of Civil Engineering, American University of Sharjah, Sharjah, United Arab Emirates.

Email: atarig@aus.edu

Received December $4^{\text {th }}, 2011$; revised January $6^{\text {th }}$, 2012; accepted January $16^{\text {th }}, 2012$

\begin{abstract}
The use of Global Navigation Satellite Systems (GNSS) for positioning has revolutionized the way location data is being collected. The NAVigation System with Time And Ranging Global Positioning System (GPS), which is a principal component of the global navigation satellite system (GNSS); is a satellite-based radio navigation system that provides positions of points of interest and time information to users. GPS positional accuracy can be improved by using differential corrections obtained through a technique called Differential GPS (DGPS), which is known to provide the most accurate positioning results. Differential correction can be applied in real time at the data collection phase or in the office, at the post-processing phase. DGPS is generally used for positioning purposes through static or kinematics GPS surveys. In static GPS surveys, one receiver is placed at a point whose coordinates are known and the other receiver is placed over a point whose coordinates are desired. In kinematic surveys, one receiver remains at one point (base station) normally with known coordinates, and the other receiver (rover) moves from point to point in the project area. Kinematic surveys in which points positions are computed on-the-fly (OTF) are known as real-time kinematic (RTK). RTK surveys provide real-time locations of points of interest needed in many applications. Positioning with wide-area GNSS networks is basically based on the DGPS and RTK concepts. Observables from a network of a finite number of GPS receivers over an area are processed by a server at a central location (network server) and made available to the users of the network later or in real-time through radio-based, satellite, or wireless communications. This article provides a review of the concept and application of positioning with wide-area GNSS networks.
\end{abstract}

Keywords: Wide-Area GNSS Network; Virtual Reference Station (VRS)

\section{Introduction}

The 3D coordinates of the GPS receiver position and the receiver clock error can be estimated based on the trilateration concept provided that at least four GPS satellites are simultaneously tracked by the GPS receiver. Trilateration is a geometric method used to determine the location of a point using the geometry of spheres based on distance measurements [1]. The position and velocity vectors of each satellite can be acquired from the broadcast ephemerides. Much precise ephemerides can be obtained from the International GPS Services (IGS). The positioning accuracy can be improved with more observations either from other satellites that are simultaneously tracked or from the same set of satellites with longer observing time. The distance from a GPS receiver to a satellite (called range) can be obtained from two GPS observables: 1) pseudo ranges (from codes) and 2) phase ranges. The pseudo range observable is a measure of the distance between the satellite and the receiver's antenna, referring to the epoch of emission and reception of the codes [2]. The range can be determined by multiplying the speed of light and the total travel time, which is inferred from correlateing the identical pseudo-random noise (PRN) of the received codes to the receiver-generated replica. On the other hand, the range can also be expressed by the total number of waves, including the integer and the fractional parts, multiplied by the wavelength of the carrier wave [3]. The phase observable is the fractional part of the phase difference between the received wave and that of the internal receiver oscillator. The integer part of the exact number of carrier waves from each satellite to the antenna, called the initial integer ambiguity, remains unknown and needs to be solved for. The correct ambiguity solution is a key to achieve higher accuracy in the kinematic GPS positioning. It is common to use both code and phase observations, provided that the receiver is equipped with such capabilities. A typical single commercial GPS receiver (non-geodetic grade) can estimate the user's position to within an average accuracy of about 12 meters, 
which is not satisfactory for many applications. In fact, GPS can produce positions that are accurate to sub-centimeter level. Many factors affect the accuracy of GPS positioning including orbital errors, satellite and receiver clock errors, signal propagation errors, measurement noise, satellite geometry measures, tropospheric delay, ionospheric delay, multipath errors, ect..

GPS positional accuracy can be improved by using differential corrections obtained by using a technique called Differential GPS (DGPS), which has been known to provide the most accurate positioning results [4]. Differential correction can be applied in real time at the data collection phase or in the office, at the post-processing phase. The simple setting of the Differential GPS (DGPS) involves the cooperation of two receivers: one is referred to the base or reference station (whose coordinates are known with the associated variance and covariance); the other is referred to the rover (whose coordinates are to be determined while being either stationary or moving). With both receivers collecting data simultaneously, the common errors such as the clock synchronization errors and the atmospheric range delays can be eliminated by differencing the observations of both receivers with respect to the same set of satellites. DGPS provides the rover's coordinates with respect to the reference station due to the differencing; that is, the $3 \mathrm{D}$ coordinate components of the relative position vector from the reference station to the rover's position. In addition, as the baseline length between the reference station and the rover's position increases, the number of satellites simultaneously tracked decreases, and therefore the atmospheric conditions at the ends of the baseline start to de-correlate [5]. As a result, the differencing can no longer eliminate as much atmospheric errors as it would in the case of shorter baseline. Hence, the rover's position accuracy depends on that of the reference station's coordinates as well as the baseline length. The length of the baseline between the base station and the location to be estimated affects the positioning accuracy as shown below:

DGPS positioning accuracy $= \pm[0.5 \mathrm{~cm}+1 \mathrm{ppm}$ of the baseline length]

This demonstrates that DGPS is largely dependent on the length of the baseline. This limitation can be resolved by using shorter baselines or by establishing a so-called virtual reference station (VRS). The concept of VRS will be covered later in the paper. Although DGPS reduces atmospheric, ephemeris, and satellite clock errors; it doesn't reduce errors form satellite measurement geometry, multipath, or the receiver. Nevertheless, networks of points based on DGPS concept are being established by governmental and nongovernmental organization around the world for positioning and navigation purposes. These DGPS networks whether used for positioning or navigation, are commonly known as wide-area GNSS networks.
A GPS receiver location within a wide-area GNSS network can be estimated based on network location's server technology adopted to resolve the receiver's location. This type of technology is being used to support the United States Federal Communication Commission's Enhanced 911 requirement for public safety location services, which is commonly known as E911. DGPS is generally used for positioning purposes through static or kinematics GPS surveys. In static GPS surveys, one receiver is placed at a point whose coordinates are known and the other receiver is placed over a point whose coordinates are desired. In kinematic surveys, one receiver remains at one point (base station) normally with known coordinates, and the other receiver (rover) moves from point to point in the project area. Kinematic surveys in which points positions are computed on-the-fly are known as real-time kinematic (RTK).

Unlike static DGPS surveys, which is often used for precise control surveys, RTK surveys provide real-time locations of points of interest that are typically needed in many engineering applications such as setting up project control, construction layout, topographic mapping, asbuilt surveying, ect.. RTK surveying requires both the reference and remote receivers to simultaneously acquire dual-frequency (L1/L2) GPS observations and perform carrier phase integer ambiguity resolution. Specifically, a fixed solution is essential for RTK surveys for surveying and engineering projects. It is common to initiate RTK surveys at a known point although most of GPS systems adopt an On-The-Fly (OTF) initialization technology, which uses the L2 frequency. In this case, the rover can initialize and resolve integers without a period of static initialization. The advantage of the OTF ability is that if a loss of satellite lock occurs, initialization can occur while in motion and the integers can be resolved OTF at the rover within no more than 30 -seconds. This is why a communication link is required between the two receivers in order to obtain the real-time coordinates of the rover's location [6]. This communication link is normally established through radio, cell phone, or satellite. Being a typical DGPS method, the problem with the common RTK is that errors especially atmospheric; increase with the increase of baseline length. This is a big issue in a traditional RTK network because a dense network of reference stations is required to maintain shorter baseline lengths. This is not an issue with wide-area GPS network since now the network GPS data is acquired at network server, which then processes the data, computes corrections, and sends the corrections to the rover, which can then estimates its position robustly.

\section{Wide-Area GNSS Network Concept}

Positioning with wide-area GNSS networks is basically 
based on the DGPS and RTK concepts. Observables from a network of a finite number of GPS receivers over an area are processed by a server at a central location (network server) and made available to the users of the network later or in real-time through radio-based, satellite, or wireless communications. If the GPS corrections are communicated to the user in real-time, then the wide-area network is known at real-time network (RTN). The central server computes orbital, clock, and atmospheric errors over the GPS network and broadcasts the corrections to the users through one of the means mentioned above in real- or delayed-time. Radio-based communication between the master station and the network receivers requires a clear line-of-sight and short baseline distance, which cannot be practically guaranteed in every survey [7]. This problem with radio-based communication inspired the relatively new wireless-based communication. Depending of the length of baseline, positional accuracy of target points within a wide-area network is in the range 5 - 10 centimetres horizontally and is in the range 15 - 20 centimetres vertically for real-time positioning. The main objective of a RTN is to provide positioning and/or navigation service continuously with a quality level that meets users requirements. The quality of the user's position estimated within the RTN is a function of many variables including the stations spacing (related to length of baseline(s)), network server configuration, error model, redundancy of communication between rover and master station server, broadcast format (RTCM1.0, 2.3, 3.1, $\mathrm{CMR}+$, ect.), and network logistics (software, hardware, power sources, backup capability, ect.).

In this section, the concept of wide-area GNSS network will be illustrated and a few positioning networks will be presented. Currently, many wide-area GNSS networks exist around the world, including over a hundred networks in the US alone; ranging from publically to privately developed and operated. Some of these networks are GNSS and some are GPS only networks. Example of a wide-area system that is only DGPS (non-real-time) is the National Geodetic Survey's (NGS) Continuously Operating Reference Stations (CORS). The concept and the typical architecture of a private real-time GNSS network is presented in this article along with the virtual reference station (VRS) concept, which is being used largely in private networks. Not presented in this paper are the wide-area systems that are used solely for navigation purpose. Example of such a network system is the US Federal Aviation Administration's (FAA) Wide Area Augmentation System (WAAS) [8].

\subsection{Continuously Operating Reference Stations (CORS)}

Many CORS networks have been developed by a number of governmental, private, and academic organizations to provide support for precise positioning and navigation for applications ranging from ordinary engineering work, to robust target location identification, and scientific research. For illustration purposes, this section presents the National Geodetic Survey (NGS) CORS network, which covers the United States. CORS is an active wide-area GNSS network managed by the National Geodetic Survey (NGS) in cooperation with the United States Coast Guard, Federal Aviation Administration, and US Army Corps of Engineers, and it offers DGPS service to the public free of charge. The development of CORS was motivated by the accuracy requirements for many activeties in surveying, mapping, engineering and related fields. In CORS network, there are always two sets of stations: 1) network stations, which are precisely known locations within the network, which continuously collect GPS data, and 2) user stations; fixed or mobile, which are unknown or approximate locations. The data collected at the network stations are used to compute GPS corrections for the network including the user stations within the network area. The user commonly receives these corrections after sending the GPS data file to NGS.

A typical CORS network master control facility collects, stores, and processes GPS data acquired for the stations within the network. The GPS coordinates of unknown (new) points are then estimated based on data collected at a CORS station and processed by the master CORS facility based on the relative positioning concept [9]. Besides being utilized for relative positioning, some reference stations within CORS network depending on its location and other specific characteristics may be used by NGS and other governmental agencies in the US for clock correction, precise ephemerides, crustal motion monitoring and atmospheric and earth rotation studies [10]. NGS provides the coordinates of CORS stations in both of North American Datum of 1983 (NAD83-2002_epoch) and The International Reference Frame of 2000 (ITRF00), which has the same ellipsoid's scale and shape parameters of the well-known World Geodetic System of 1984 (WGS84).

GPS data collected for a time period of up to a fullobservation-day is transferred from a CORS site to the master station. Once the GPS data from the CORS station is received by the master station, it gets processed to generate files of same format that are sent back to the user and sometimes archived for further processing. NGS also makes precise ephemeris data available to the GPS user community as it computes precise orbits based on orbital data acquired at the six GPS master stations. Each orbit covers a one-day period at a 0.25 -hour epoch including satellite clock offsets. Currently CORS data is not available in real-time streaming mode, but NGS is planning to broadcast data from some CORS stations in the near future. NGS is now supporting international and federal agencies through near real-time GPS data steam- 
ing through the International Global Navigation Satellite Systems (GNSS) Service known as IGS [11].

There are many requirements need to be met before a given site can be chosen as a CORS station. These include: location, availability of basic utilities such as power source and backup, continuous network connection, ease of mounting the GPS receiver and peripheral equipment, adequate sky visibility from the CORS antenna location, availability of cable and/or telephone line, climate control capability, and security and ease of access. In wide-area networks, the GPS receiver type, model, software, data acquisition rate, ect.; is the backbone of the positioning or navigation process. An ideal CORS receiver should be able efficiently collect the pseudo-ranges and the two carrier phases of the P- and CA-codes [12].

The major benefit of CORS network is the ability of the GPS user to acquire his/her unknown position within the CORS network by performing relative positioning with no need for a base station. At a CORS station, NGS provides the GPS coordinates based on L1 phase center as well as the Antenna Reference Point (ARP). The ARPbased coordinates are those estimated based on the bottom mount of the GPS antenna from which the phase center offsets are computed. For precise positioning using CORS network, the post-processed orbital GPS data can be obtained through the internet or wireless connections from NGS. One major issue with CORS-based positioning is quality control in the context of the utilized CORS station. These issues are mainly related to multi-path errors, receiver errors, cycle slip errors, issues with the positional stability of the GPS antenna, ect.. Although monitoring of the positional stability of the GPS antenna can be carried out by repeated inter-station baseline calculations, it is rarely considered a serious issue by the users. All of these issues have to be addressed by the GPS user locally; otherwise the estimated GPS coordinates will have low quality.

\subsection{Private Wide-Area Real-Time GNSS Networks}

The demand for precise GPS data in the surveying, engineering, mapping, and related professions is continuously increasing to meet the need for spatial data for lots of applications especially in areas that have witnessed a boom in real estate businesses. Traditionally, radio-based RTK has been known to provide the most accurate positioning as it corrects the inherent errors of the satellite system at the location of a roving GPS receiver. Issues with radiocommunication that relate to clear line-of-sight between the locations of the base station and the rover as well as short baselines inspired the development of more reliable communication mechanism. The latest communication techniques utilize wireless communication through ultrahigh-frequency "UHF" radio, satellite, or cellular phone modems. This section presents the concept and the applications of privately operated wide-area GNSS network; specifically a wireless-based network.

\subsubsection{Working Principle of Private Wide-Area Real-Time GNSS}

GPS observation data from a set of fixed reference stations is continuously transmitted to a central server. The network processor performs integrity checks on all GPS observables, carries out quality checks on the data, and correct for cycle slips. The network processor resolves the network ambiguities and computes atmospheric corrections based on double-differencing concept after GPS data integrity has been checked. Figure 1 below shows an example of the general architecture of the wirelessbased RTK network.

\subsubsection{Wireless-Based Private Wide-Area Real-Time GNSS Network}

Wireless-based GNSS network is a wide-area, real-time integrated GPS positioning system, consisting of GPS hardware, software and wireless communication links. The communication links such as ultra-high-frequency "UHF" radio, satellite, or cellular phone are utilized by the network to provide communication between the network server and the reference stations to model errors throughout the coverage area. This model is used; for example, to create virtual reference stations (VRS) near the user's location which then provide a localized set of standard format correction messages to the rover. To enable the GPS modeling, the rover provides its approximate position to the central server via a cellular modem. The central server processes double-differenced data, generates errors, creates a VRS, and interpolates and applies corrections for the rover. The central server then produces corrections as if they were coming from the VRS and transmits them back to the receiver through the communication links. This type of a wide-area network is

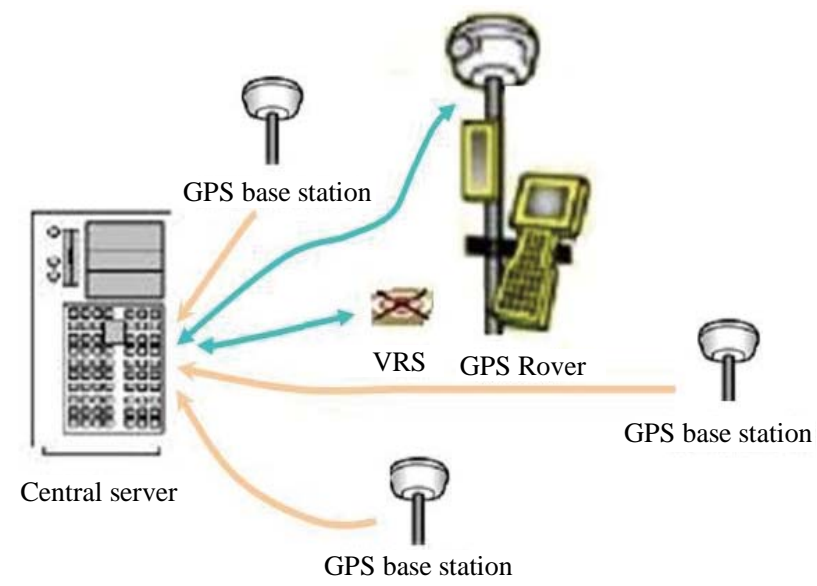

Figure 1. Example of the general architecture of a wirelessbased wide-area GNSS network. 
managed by software packages available from; for example Trimble ${ }^{\circledR}$ Navigation, including GPS BASE, GPS NET, and RTK NET. GPS BASE provides continuous, fully automatic operation of a single base station for post processing of RTK or DGPS corrections within a local area. GPS NET is designed to connect multiple base stations. RTK NET uses real-time data from the GPS NET software to generate corrections for high accuracy [13], real-time Kinematic (RTK) corrections throughout the network. RTK NET is the software that provides the Virtual Reference Station (VRS) broadcast corrections.

The VRS is the oldest and one of five RTK wide-area GNSS network correction methods including the FlächenKorrektur Parameter (FKP), the Individualized MAC (i-MAC), the Master Auxiliary Corrections (MAC), and the Networked Reference System (NRS). Conceptually, the VRS and i-MAC methods are not different, but the i-MAC method generates corrections for a real station and doesn't create a VRS. Unlike the VRS and i-MAC, the FKP method creates corrections in terms of simple planes for a finite area around the closest reference station to the rover. Also, the FKP method does not require the rover to send its approximate position; instead, the server sends the GPS data along with a distance-based error model of the reference station to the rover [14]. The corrections estimated by the server at the rover's position in this method are based on the assumption that the distancebased errors between the references stations change linearly, which can result in low quality position estimates. In the MAC method, the server transmits the GPS data and network information to the rover, which can then calculate the corrections based on single- or multiple-baselines. The advantage of this method is that the rover can recalculate its position OTF with the RTK solution, but the disadvantage is that the corrections are computed by the rover at the user end; therefore the type, brand and quality of the receiver becomes an issue. Networked Reference System (NRS), is the newest RTK method and it uses a sub-set of the network reference stations within a finite area around the rover location to generate the error model and it computes corrections for long baselines (up to 200 kilometres).

The VRS concept is mainly based on the elimination of the residual double differenced errors for long-range baselines between known and unknown stations, and an optimum set of GPS data is selected from a combined observations from several reference stations. Accordingly, the methods for short-range baseline kinematic data processing can be used to determine the position of a long-range rover based on the VRS data. In order to create a VRS near the receiver, approximate position must be resolved at first from the observations of multiple stations in the network. Then, the corrections for the rover location can be generated from the residuals in carrierphase measurements, and accordingly corrections are computed for the rover approximate position.

\subsubsection{Benefits of a Private Wide-Area Real-Time GNSS}

A private wide-area real-time GNSS network makes the determination of coordinates more accurate, much easier, and more efficient compared to traditional methods. Such capability enables for real-time, efficient GPS data collection and accurate positioning needed in many applications including surveying, GIS, utility mapping, natural resources, environmental monitoring, transportation engineering, ect. [15]. Another emerging mapping method that uses a continuously operating GPS network to improve mapping accuracy is mobile mapping where the mapping equipment is connected to a roving GPS unit. A new mobile mapping application in transportation engineering utilizes mobile data terminals installed in emergency vehicles, public local transportation and transit systems based on a GPS tracking system. If such a tracking system uses a wide-area real-time GNSS network, the efficiency of positioning and public safety will be improved. In summary, wide-area real-time GNSS network increases the positioning productivity, simplify data acquisition process, increase the accuracy, and allow for rapid and easy GPS equipment set-ups.

\section{Concluding Remarks}

Evidently there are many advantages of a wide-area GNSS network that include, but not limited to no need for a user base station, orbital and atmospheric errors are estimated at the user's location based on a network model, and ease of GPS equipment set-ups that would result in higher productivity. In the case of a RTN however, the communication links between the network server and the rover remain an issue when wireless or radio broadcasts are lost. For example, this is not unusual scenario with wireless connections, which would not be available in some remote areas, or radio broadcast which basically depends on the length of the baseline and the clarity of the line of sight. This later scenario becomes obvious when positioning is sought outside the RTN envelope resulting in coordinate estimates with lower quality. Another issue that applies not only to the RTN, but to all GNSS products is related to interference and jamming of the GPS signal, especially interference which can take place due to increased solar activity or bad weather. Another issue that worth mentioning is related to the quality of positions estimated based on these wide-area GNSS networks. This is the lack of standards, which applies not only to wide-area GNSS networks positions, but to every spatial data product. Technically, spatial data standards do exist, however there are only used as guidelines be- 
cause the enforcement of the standards in this case is not practically feasible. In an effort to provide standards for NGSS networks-based positioning, NGS has recently released guidelines for RTN networks; specifying the requirements from the GPS survey planning phase, to the establishment and the operation phases. The development of these guidelines was motivated in part by the need for general standards for a rapidly growing nationwide infrastructure of more than a hundred wide-area GNSS networks. The release of the guidelines is also essential because coordinates estimated within these networks are commonly based on US national datums, which need to be in harmony with the National Spatial Reference System (NSRS).

\section{REFERENCES}

[1] G. Seeber, "Satellite Geodesy,” Walter de Gruyter, New York, 1993.

[2] A. Leick, “GPS Satellite Surveying," 2nd Edition, Wiley, New York, 1994.

[3] R. Langley, “The GPS Observables,” GPS World, Vol. 4, No. 4, 1993, pp. 55-69.

[4] P. Bolstad, A. Jenks, A. J. Berkin, K. Horne and W. Reading, "A Comparison of Autonomous, WAAS, Real-Time, and Post-Processed Global Positioning Systems (GPS) Accuracies in Northern Forests," Northern Journal of Applied Forestry, Vol. 22, No. 1, 2005, pp. 5-11.

[5] C. Gaod, "Short Distance GPS Models, in GPS for Geodesy,” 2nd Edition, In: P. Teunissen and A. Kleusberg, Eds, Springer-Verlag, Berlin, 1998.

[6] J. Sickle, "GPS for Land Surveyors," 3rd Edition, CRC Press, Taylor and Francis Group, New York, 2008.
[7] C. Kee, “Wide Area Differential GPS,” In: B. W. Parkinson and J. J. Spilker Jr., Eds., Global Positioning System: Theory and Applications, American Institute of Aeronautics and Astronautics, Inc., Washington DC, 1996, pp. 81-116.

[8] Federal Aviation Administration, "Current WAAS Vertical Navigation Service Snapshot Display,” 2011. http://www.nstb.tc.faa.gov/RT_VerticalProtectionLevel.h tm

[9] National Geodetic Survey, "Location Map of the CORS Stations,” 2011. http://www.ngs.noaa.gov/CORS

[10] P. Spofford, "GPS CORS and Precise Orbit Data from the National Geodetic Survey,” 1996. http://www.ngs.noaa.gov/PUBS_LIB/GPSCORS.pdf.

[11] National Geodetic Survey, "Guidelines for Real-Time GNSS Networks,” NOAA's National Geodetic Survey Publication Library, 2011. http://www.ngs.noaa.gov

[12] M. Leach and M. Cardoza, "GPS Monitor Stations, Reference Stations, and Networks: Design Issues and Trends in Technology," International Federation of Surveyors, Melbourne, 1994. http://www.tridentresearch.com/pubs/papr6.pdf

[13] L. Arnold and P. Zandbergen, "Positional Accuracy of the Wide Area Augmentation System in Consumer-Grade GPS Units,” Computers \& Geosciences, Vol. 37, No. 7, 2011, pp. 883-892. doi:10.1016/j.cageo.2010.12.011

[14] G. Wübbena, A. Bagge and M. Schmitz, "Network-Based Techniques for RTK Applications," Proceedings of the Japan Institute of Navigation, GPS Symposium, Japan Institute of Navigation, Kobe, 2001, pp. 53-65.

[15] eGPS Incorporation, "Current WAAS Vertical Navigation Service Snapshot Display,” 2011. http://egps.net/ 